\title{
High-grade undifferentiated pleomorphic cardiac sarcoma: A rare malignant primary cardiac tumor mimicking double mitral lesion
}

\author{
Sarcoma cardíaco pleomórfico indiferenciado de alto grado: un tumor cardíaco maligno \\ primario que simula una doble lesión mitral
}

\author{
Juan C. Espinoza-Cobos ${ }^{1}$, Sergio Ortiz-Obregón ${ }^{1}$, Enrique Blanco-Lemus², Gerardo Gutiérrez-Tovar ${ }^{1}$, \\ Luis E. Santos-Martínez $z^{3}$ and Luis A. Moreno-Ruiz * \\ ${ }^{1}$ Surgical Intensive Care Unit; ${ }^{2}$ Department of Pathology; ${ }^{3}$ Department of Pulmonary Hypertension Clinic and Right Ventricle; ${ }^{4}$ Adult Valvular, \\ Degenerative and Congenital Heart Diseases. Hospital de Cardiología, Centro Médico Nacional Siglo XXI, IMSS, Mexico City, Mexico
}

The high-grade undifferentiated pleomorphic cardiac sarcoma (UPS) is the second most common primary malignancy in the heart; usually remains asymptomatic, until they produce a mass effect or embolization. A 47-year-old female previously healthy was admitted for a 7-day history of progressive dyspnea. At physical examination, there was mitral regurgitant murmur Grade III and bilateral crepitation rales; the electrocardiogram detected sinus tachycardia (Fig. 1A) and X-ray showed Grade II venocapillary hypertension and pleural effusion (Fig. 1B). The echocardiogram revealed an intracardiac mass in the left atrium of $36 \mathrm{~mm} \times 48 \mathrm{~mm}$, protruding through the mitral valve, conditioning moderate stenosis and severe insufficiency (Fig. 1C-E). Surgical treatment was realized with resection of the tumor, partial resection of the posterior wall of both atrial and interatrial septum, and mitral commissuroplasty. The histological sections showed a solid-appearing neoplastic proliferative lesion with lax isolated areas of myxoid appearance, short cellular bundles, and branched vessels with extensive myocardial infiltration and infiltration of the valvular tissue (Fig. 2A-C). Thoracoabdominopelvic tomography showed no evidence of metastatic disease. Treatment was started with chemotherapy, but she had progressive deterioration and died 3 months later. Cardiac sarcoma has an incidence of $\sim 1.7 \%$ of cases; survival at 10 years is $90 \%$ in low-grade sarcomas, $60 \%$ with intermediate grade, and $20 \%$ with high degree 1 . The treatment is surgical resection followed by chemotherapy ${ }^{2}$, however, the invasive nature of UPS makes it difficult to obtain surgical margins without tumor activity, associated with relapse and poor short-term prognosis ${ }^{3}$.

\section{Funding}

None.

\section{Conflicts of interest}

The authors declare no conflict of interest. license (http://creativecommons.org/licenses/by-nc-nd/4.0/).

Available online: 02-07-2021 Arch Cardiol Mex (Eng). 2021;91(3):388-390 www.archivoscardiologia.com
an open access article under the CC BY-NC-ND 


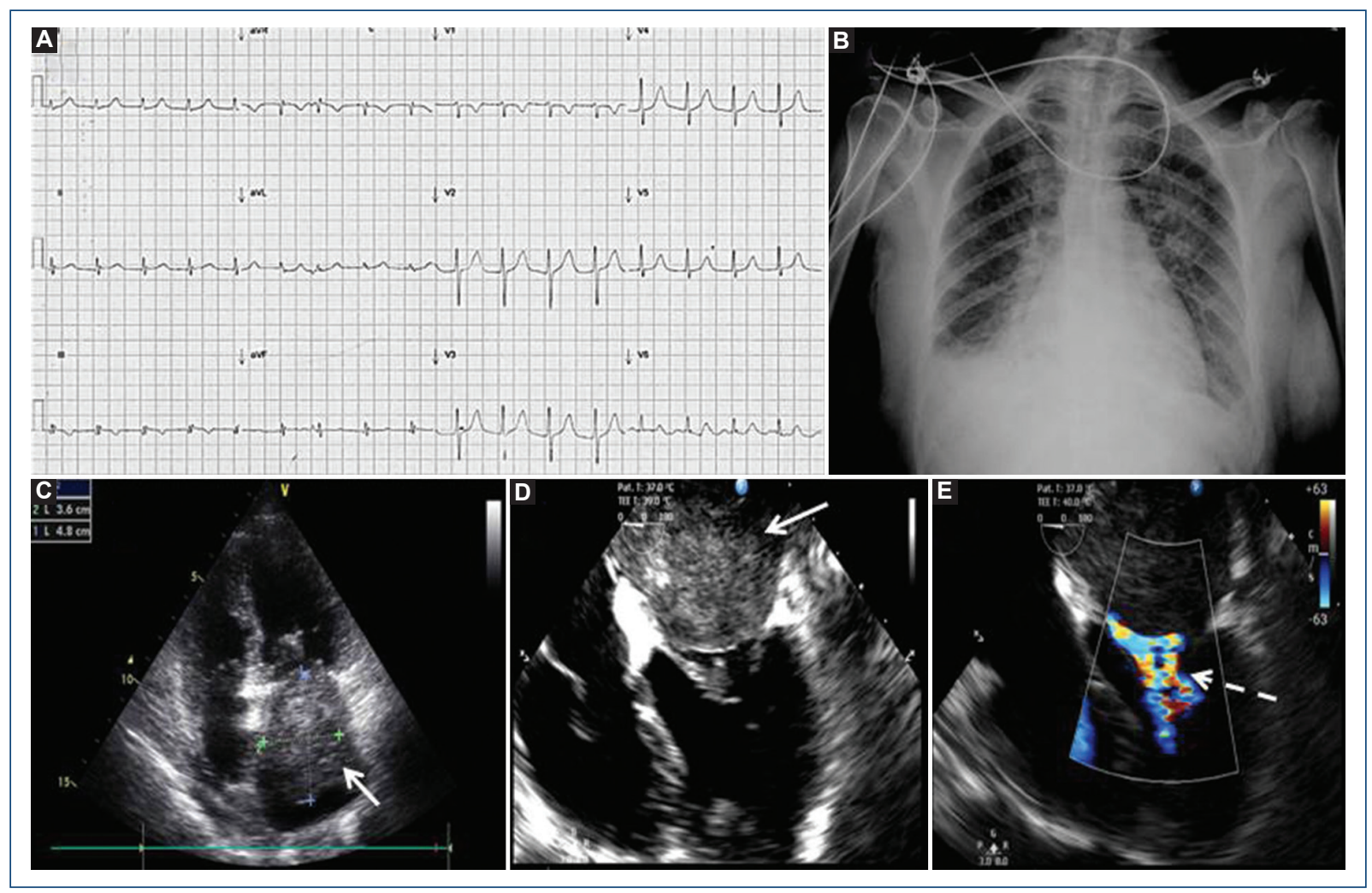

Figure 1. Electrocardiogram and multimodality imaging approach of undifferentiated pleomorphic cardiac sarcoma. A: 12lead electrocardiogram shows sinus tachycardia; B: chest X-ray with Grade II venocapillary hypertension and pleural effusion; C: four chambers image by transthoracic echocardiogram, showing intracardiac mass (arrow) located in the left atrium; D: transesophageal echocardiogram confirms mass in the left atrium (solid arrow) adhered to the posterior wall of $48 \mathrm{~mm} \times 34 \mathrm{~mm}$ and E: Doppler color showed obstruction of the left ventricle inflow tract (dashed arrow).

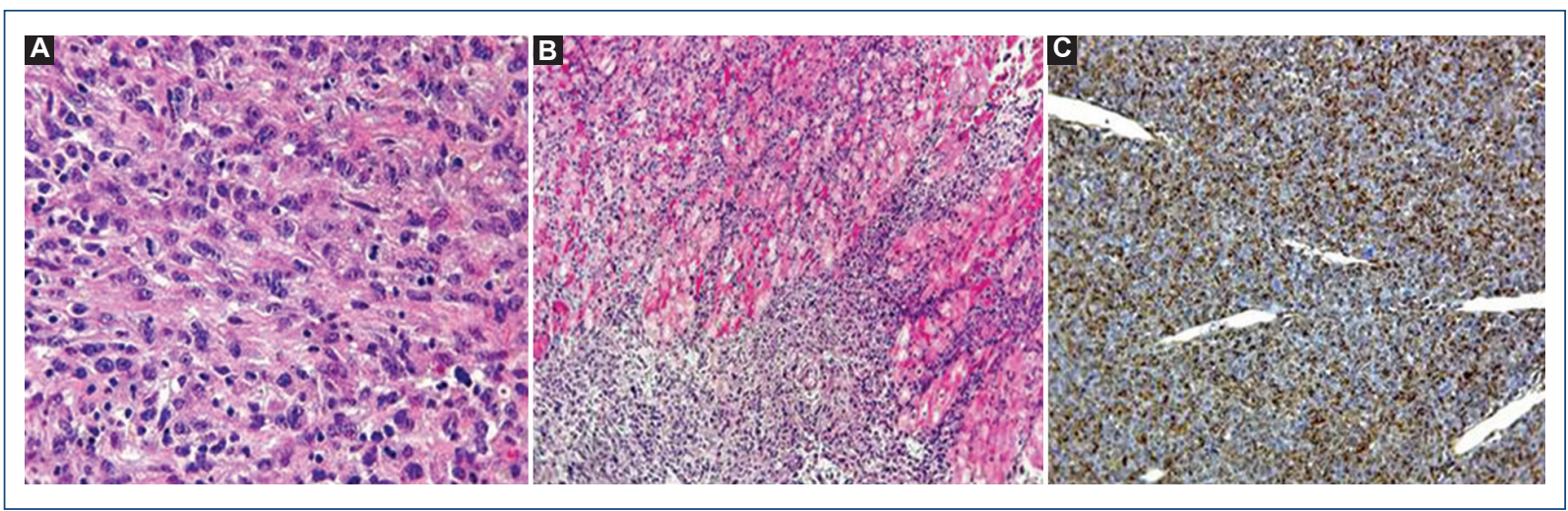

Figure 2. Histological sections of undifferentiated pleomorphic cardiac sarcoma. A: the neoplastic cells have an epithelioid or spindle cell appearance with pleomorphic nuclei, atypical mitosis, and scarce lymphocytic infiltrate (HE, $400 \times$ ); B: extensive myocardial infiltration is shown by pleomorphic neoplasm with a solid pattern (HE, $100 \times$ ); C: immunohistochemical staining with positive cytoplasmic anti-vimentin antibody (ocher color) in most of the neoplastic cells $(100 \times)$. HE: Hematoxylin-eosin. 


\section{Ethical disclosures}

Protection of human and animal subjects. The authors declare that no experiments were performed on humans or animals for this study.

Confidentiality of data. The authors declare that they have followed the protocols of their work center on the publication of patient data.

Right to privacy and informed consent. The authors have obtained the written informed consent of the patients or subjects mentioned in the article. The corresponding author is in possession of this document.

\section{References}

1. Ostrowski S, Marcinkiewicz A, Kosmider A, Jaszewski R. Sarcomas of the heart as a difficult interdisciplinary problem. Arch Med Sci. 2014;10:135-48 2. Isambert N, Ray-Coquard I, Italiano A, Rios M, Kerbrat P, Gauthier M, et al. Primary cardiac sarcomas: a retrospective study of the French sarcoma group. Eur J Cancer. 2014;50:128-36.

3. Li H, Yang S, Chen H, Yang Z, Hong T, Hou Y, et al. Survival after heart transplantation for non-metastatic primary cardiac sarcoma. J Thorac Cardiovasc Surg. 2016;11:145. 